\title{
Setting up a specific surveillance system of community health during mass gatherings
}

\author{
T Hanslik, P Y Boelle, A Flahault
}

During mass gatherings such as those that occur during international fairs or sporting events, there are no recognised standards for providing health surveillance of the general community (in contrast with the participant community). Data must be collected continuously as produced, so as to provide immediate information and permit prompt detection of epidemics. To monitor the effects of the 1998 World Football Cup (WFC) on the health of the general population of the host country, we set up a nationwide automated sentinel disease surveillance system. Its results have been published in detail elsewhere. ${ }^{1}$ We describe here the implementation of the general practitioners' (GPs) sentinel system required by this surveillance system.

\section{Methods}

Surveillance lasted two months (June and July 1998). Our protocol required that physicians communicate daily with the host computer, via an internet web site or a videotext terminal. The single page format included the total daily number of data for 11 items relating to the level of community medical activity, and to communicable, societal and environmental diseases. ${ }^{2}{ }^{3}$

GPs were recruited for the programme on a voluntary basis, throughout France, from the Sentinel General Practitioners; system (SGPs), ${ }^{4}$ and by random digit dial procedures, using the telephone list of the GPs working in the 10 cities receiving WFC matches, where recruitment was intensified so as to include 3\% of these GPs (non-SGPs). Participation in the network was monitored. GPs used to communicating data (SGPs) were compared with those not used to doing so (non-SGPs), in order to find out if they differed according to the mean number of patients seen daily and the duration of participation in the study.

Réseau Sentinelles, Institut National de la Santé et de la

Recherche Médicale, Unit 444, Université

Paris 6, Paris, France (WHO Collaborating Center for Electronic

Disease Surveillance)

Correspondence to:

Dr Hanslik, INSERM Unité

444, 27 rue Chaligny, 75571

Paris Cedex 12, France

(thomas.hanslik@

apr.ap-hop-paris.fr)

Accepted for publication 23 March 2001 number of patients seen daily, or with his proximity to an event's venue within a radius of 30 kilometres of a World Cup city (see reference 1 for details). SGPs reported the same mean number of patients seen daily as non-SGPsthat is, 18 (7.66) and 17.4 (7.5) patients, respectively (not significant, Wilcoxon test). They participated for a longer period than non-SGPs, for median periods of 45 and 38 days respectively ( $p=0.0001$, Wilcoxon test). The level of medical activity reflected by the items surveyed remained stable throughout the WFC, both in the cities that hosted matches and those that did not (data not shown).

\section{Discussion}

For monitoring health issues in the general community during mass gatherings, a nationwide real time electronic active surveillance system based on sentinel physicians proved very feasible. It also proved acceptable in terms of participation time. The surveillance system was sensitive, because it would have captured one case of each disorder under surveillance with a $95 \%$ probability if at least 100 cases had been seen in general practice, according to the Poisson distribution, and because about 3\% of the GPs were represented in the WFC cities. We may assume that our sample was fairly representative of French GPs, for four reasons: (1) the participating SGPs did not differ from all SGPs (data not shown) and the characteristics of the SGPs are comparable to those of all French $\mathrm{Gps}^{5}$; (2) non-SGPs were recruited by random digit dial procedures; (3) non-SGPs did not differ from SGPs as regards the declared incidences of the surveyed items; and (4) the overall male/female ratio of the GPs participating in this study was 4, compared with 3.8 for all French GPs. Real time electronic surveillance ensured appropriate timeliness. This system could therefore serve as a model for any ongoing monitoring of general community health during international special events, or for any other short-term intensive nationwide health surveillance.

During the study period, using the same model, other surveillance systems were implemented in emergency hospital departments, private emergency community services, community health centres, and the medical centre of the Paris airports. ${ }^{1}$ Like the sentinel GPs, none of these sources detected any significant health events.

This initiative may be generalisable to countries without a sentinel surveillance system. Most of the physicians participating in the present surveillance programme had never been involved in a surveillance system and were 
not experienced in data communication. All the electronic devices used were especially created for this particular programme, and were distinct from existing computer developments. We used hardware equipment that was light (a single desktop PC), and software based on free access internet technologies. Internet access is now increasingly widespread. The expenses and resources necessary for such an operation were not excessive (marginal costs of the programme were evaluated at about US\$20000).
Funding: this work was partly funded by a joint grant, no 9808100, from INSERM and Synthelabo Laboratories.

Conflicts of interest: none.

1 Hanslik T, Espinoza P, Boelle PY, et al. Sentinel monitoring of general community health during the 1998 World Football Cup. Rev Epidemiol Sante Publique 2001;49:135-45.

2 Leonard RB. Medical support for mass gatherings. Emerg Med Clin North Am 1996; 14:383-97.

3 Wetterhall SF, Coulombier DM, Herndon JM, et al. OlymWetterhall SF, Coulombier DM, Herndon JM, et al. Olym-
pic Surveillance Unit. Medical care delivery at the 1996 pic Surveillance Unit. Medical care deliver
Olympic Games. $A M A$ 1998;279:1463-8.

4 Fourquet F, Drucker J. Communicable diseases surveillance: the Sentinel network. Lancet 1997;349:794-5. 5 Chauvin P, Valleron AJ. Attitude of French general practitioners to the public health surveillance of communicable diseases. Int f Epidemiol 1995;24:435-40.

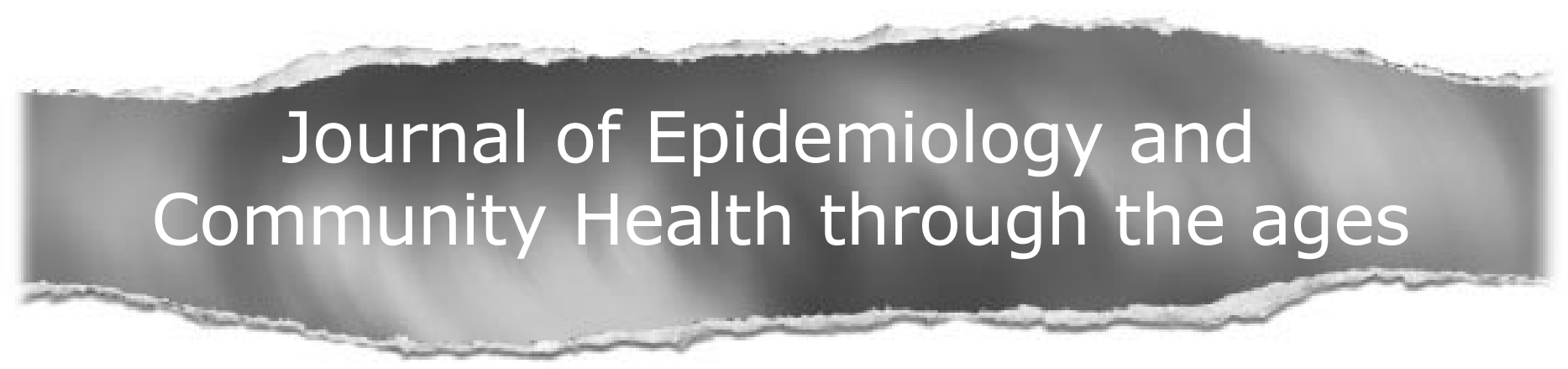

Browse the Archive

Journal of Epidemiology and Community Health online has an archive of content dating back to 1978. Full text from January 2000; abstracts from 1978; table of contents from 1978

\section{www.jech.com}

